\title{
Effects of differential diffusion on the stabilization of unsteady lean premixed flames behind a bluff-body
}

\author{
Yu Jeong Kim • Bok Jik Lee • Hong G. Im
}

Received: date / Accepted: date

\begin{abstract}
Two-dimensional direct numerical simulations were conducted to investigate the effects of differential diffusion on flame stabilization and blow-off dynamics of lean premixed hydrogen-air and syngas-air flames stabilized on a meso-scale bluff-body in a square channel. The unity Lewis number for all species was imposed to isolate the effects of differential diffusion. Four sets of simulation cases were conducted. Two different inflow temperature with unity Lewis number were applied to examine distinct levels of hydrodynamic instability. Each unity Lewis number case was compared with the non-unity Lewis number case to investigate how differential diffusion affects the overall flame responses, instabilities, and blow-off mechanism. For all cases, the overall flame dynamics were observed in several distinct modes as the inflow velocity approaches blow-off limit. One of the primary effects of unity Lewis number was an increased level of hydrodynamic instability due to the lower flame temperature and thus a lower density ratio. The lower gas temperature also led to a weakening of the re-ignition of the quenched local mixture by the product gas entrainment. The combined effects were manifested as suppression of the re-ignition events, leading to a revised conclusion that the ultimate blow-off behavior at high velocity conditions are mainly controlled by the onset of local extinction.
\end{abstract}

Keywords Direct numerical simulation · Bluff-body · Lean premixed flames · Unity Lewis number $\cdot$ Stabilization mechanism $\cdot$ Blow-off

\footnotetext{
Yu Jeong Kim · Hong G. Im

Clean Combustion Research Center, King Abdullah University of Science and Technology, Thuwal 23955-6900, Kingdom of Saudi Arabia

Tel.: + 966-12-808-4726

E-mail: hong.im@kaust.edu.sa

Bok Jik Lee

Institute of Advanced Aerospace Technology, Seoul National University, Seoul 08826, Republic of Korea
} 


\section{Introduction}

Bluff-bodies of various types have commonly been employed in premixed combustion systems in order to achieve flame stabilization over a wide range of operating conditions. The main idea is to create recirculation zones behind the solid object to increase the flow residence time and heat recuperation by the residual hot products. At highly turbulent conditions, however, the bluff-bodies also involve large fluctuations in flames and flow field such as unstable vortex shedding, which may lead to a total blow-off. Understanding the physical mechanism of flame stabilization and blow-off is important in order to ensure steady operation of the combustion devices. Due to the complexities of dynamics and many physical and chemical parameters involved, it is difficult to obtain a comprehensive understanding of the phenomena.

Shanbhogue et al. [1] reported a general review about lean blow-off process, scaling of blow-off, and dynamics of bluff-body flow/flames for both non-reacting and reacting flows in a wide range of Reynolds number conditions. Experimental studies $[2,3]$ have been conducted for non-reacting flows behind the bluff-body to understand the physical mechanism of unsteady flow in a channel. For reacting laminar flows, many studies have investigated lean blowout cases both experimentally $[4,5]$ and numerically $[6,7]$ to understand the physical mechanism. An experimental study by Nair and Lieuwen [4] reported near blow-off dynamics showing two stages of blow-off with localized extinction. Turbulent reacting flow cases were also reported on blow-off mechanisms $[8,9]$ and the effects of preferential transport [10].

Recent numerical studies have reported flame stabilization and blow-off mechanism. Kedia and Ghoniem [6] studied flame anchoring mechanism for laminar methane-air flames at a fixed low Reynolds number $\left(\operatorname{Re}_{D}=500\right)$. They investigated the role of the sharp velocity gradients near the bluff-body in flame anchoring, which contributes to the preferential diffusion effects. They showed the preferential diffusion results in a local accumulation within the recirculation zone. Another experimental and numerical studies by Vance et al. [7] reported distinct lean blow-off responses depending on the Lewis number (Le). Both studies demonstrated that a large preferential diffusion effect in the recirculation zone causes a locally enriched flame in mixtures when Le $<1$. Both studies were conducted for the lean blowout behavior at low Reynolds number conditions so that there was no effects on unstable hydrodynamic flame responses.

Our recent studies $[11,12]$ explored the other end of the spectrum where the flame extinction and reignition occur in the recirculation zone at higher flow velocities. High fidelity numerical simulations were conducted for both lean premixed hydrogen and syngas flames in an unconfined flow field. Several distinct flame modes of symmetric and asymmetric vortex shedding were observed as inflow velocity approaches blow-off limit in the overall laminar flow range. It was found that the flame modes were noticeably changed due to the enhanced fluid dynamic effects of density ratio, consistent with the observations from earlier experimental studies [5,13-16]. The fluid dynamic effects were found to be an important factor in determining the ultimate blow-off limit, and the mode was properly described by the Strouhal number scaling. The Damköhler number based on the flow residence time and the ignition delay time inside the recirculation zone was found to be a good metric in predicting the blow-off limit, supporting the classic theory 
by Zukoski and Marble [17]. However, these studies [11,12] employed lean hydrogen/air mixtures, raising questions about the effects of the preferential diffusion modifying the thermal and composition conditions in the extinction/reignition regions.

Therefore, the objective of the present study is to extend our previous work to further investigate the differential diffusion effects on the flame stabilization and blow-off dynamics at higher velocity conditions. To isolate the differential diffusion effects, simulations were conducted in pairs by imposing a unity Lewis number assumption for both hydrogen-air and syngas-air mixtures so that the results are directly compared with our previous studies $[11,12]$ with realistic molecular transport $($ Le $<1)$. The effect of unity Lewis number was examined in two distinct conditions of hydrodynamic instability, which are two distinct density ratios (approximately 5.0 and 3.3) for each mixture, by preheating the inflow temperature. Continuing on our previous work, a key question to investigate is how Lewis number affect blow-off and flame stabilization mechanism, i.e. is the blow-off a result of flame quenching or failure to reignite? Comparison of non-unity and unity Lewis number flames are conducted in order to provide answers to this question, as the flame approaches the blow-off limit after undergoing the sequence of instability modes as the inflow velocity is increased continually. Lastly, detailed time scale analysis is examined to identify physical mechanism of flame stabilization and blow-off dynamics.

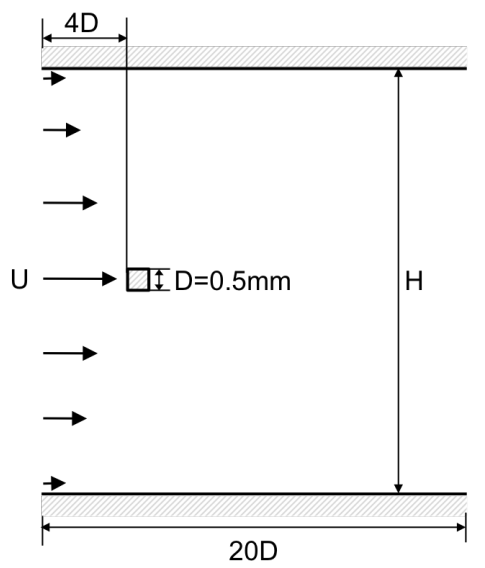

Fig. 1: Computational configuration of a two-dimensional channel with a square-shape bluffbody stabilizer.

\section{Numerical method}

Two-dimensional direct numerical simulations (DNS) solve fully compressible multispecies reacting Navier-Stokes equations with a finite difference method using the eighth order in space and fourth order in time [18]. A square channel of $10 \mathrm{~mm}$ 
$(H)$ was used as a computational domain with a square-shaped bluff-body stabilizer of $0.5 \mathrm{~mm}(D)$, whose leading edge is located at $4 D$ downstream of the inflow boundary, as shown in Figure 1 . The domain size $(H)$ was chosen to minimize the wall confinement effects on the flow field. The convergence test was done as varying blockage ratio $(D / H)$ until convergence was reached for the Strouhal number $(\mathrm{St}=f D / U)$ of the vortex shedding for the non-reacting condition [19]. The blockage ratio for this study is 0.05 .

Chemical Kinetics, thermodynamics and transport properties are evaluated using CHEMKIN libraries [20]. For the new simulations in the present study, thermal conductivity is approximated by the simplified relation as a function of temperature [21]. The mass diffusion coefficients for all species are determined by constant Lewis numbers and Prandtl number. The unity Lewis number for all species are applied in the present simulations to isolate differential diffusion effects. The simulation data for detailed transport model are taken from our previous study [11]. For the chemical reaction, detailed mechanisms of hydrogen-air and syngas-air, consisting of 9 species and 19 reactions [22] and 12 species and 33 reactions [23], respectively, are adopted. A Cartesian grid system with a uniform spacing of $\Delta x=\Delta y=10 \mu \mathrm{m}$ is chosen. A typical run with a Cray XC40 cluster (Shaheen II) for time integrations up to $10 \mathrm{~ms}$ took approximately 5,200 and 7,200 CPU hours, for hydrogen-air and syngas-air mixture, respectively.

Table 1: Inflow quantities for lean premixed hydrogen-air and syngas-air mixtures at $\Phi=0.5$ and $T_{\mathrm{in}}=300 \mathrm{~K}$ for non-unity [11] and unity Lewis number.

\begin{tabular}{c|c|c|c|c}
\hline Mixture & \multicolumn{2}{|c|}{ Hydrogen-air } & \multicolumn{2}{|c}{ Syngas-air } \\
\hline Case & H1 & H1U & S1 & S1U \\
\hline$S_{\mathrm{L}}[\mathrm{m} / \mathrm{s}]$ & 0.49 & 0.76 & 0.30 & 0.35 \\
$\rho_{u} / \rho_{b}($ Equilibrium $)$ & 4.91 & 5.01 & 5.15 & 5.20 \\
$\mathrm{Le}_{\mathrm{H}_{2}}\left(\lambda / \rho / D_{\mathrm{H}_{2}, \mathrm{~m}} / c_{p}\right)$ & 0.39 & 1.00 & 0.34 & 1.00 \\
\hline
\end{tabular}

Table 2: Inflow quantities for lean premixed hydrogen-air and syngas-air mixtures at $\Phi=0.5$ and $T_{\mathrm{in}}=500 \mathrm{~K}$ for non-unity [12] and unity Lewis number.

\begin{tabular}{c|c|c|c|c}
\hline Mixture & \multicolumn{2}{|c|}{ Hydrogen-air } & \multicolumn{2}{c}{ Syngas-air } \\
\hline Case & H3 & H3U & S3 & S3U \\
\hline$S_{\mathrm{L}}[\mathrm{m} / \mathrm{s}]$ & 2.02 & 2.04 & 0.99 & 1.01 \\
$\rho_{u} / \rho_{b}$ (Equilibrium) & 3.26 & 3.31 & 3.38 & 3.41 \\
$\mathrm{Le}_{\mathrm{H}_{2}}\left(\lambda / \rho / D_{\mathrm{H}_{2}, \mathrm{~m}} / c_{p}\right)$ & 0.39 & 1.00 & 0.34 & 1.00 \\
\hline
\end{tabular}

Non-reflecting Navier-Stokes characteristic boundary conditions (NSCBC) [24, 25] are applied to the inflow and outflow boundaries. A fully developed parabolic velocity profile with a mean value of inflow velocity $(U)$ is imposed at the inflow boundary. No-slip and adiabatic conditions are imposed at the channel walls and 
bluff-body surfaces. Lean premixed flames in hydrogen-air and syngas-air $\left(\mathrm{CO}: \mathrm{H}_{2}\right.$ $=1: 1)$ mixtures with an equivalence ratio $(\Phi)$ of 0.5 are investigated. The inflow quantities of both non-unity and unity Lewis number flames for the inflow temperature $\left(T_{\text {in }}\right)$ of $300 \mathrm{~K}$ and $500 \mathrm{~K}$ are summarized in Table 1 and 2, respectively. Case 1 and 3 represent detailed transport cases following the notation in the previous studies $[11,12]$. Case $1 \mathrm{U}$ and $3 \mathrm{U}$ are unity Lewis number cases. The inflow condition in all cases is set at pressure of $1 \mathrm{~atm}$. In this study, Lewis number (Le) of all species is artificially fixed as unity. For syngas-air mixture, volume-based effective Le is 0.8 for both cases.

For the case at $T_{\mathrm{in}}=300 \mathrm{~K}$ shown in Table 1 , unity Lewis number flame has higher laminar flame speed and similar density ratio, compared to that with the detailed transport model [11]. As our previous studies [11,12] reported, flame modes were noticeably changed due to the enhanced fluid dynamic effects of density ratio and laminar flame speed did not affect either overall flame mode or blow-off limit regarding Reynolds number $\left(\operatorname{Re}_{D}\right)$ based on the bluff-body size $(D)$. Therefore, flame tendency controlled by fluid dynamic effects of density ratio over $U$ would not be changed. At each increment of the mean inflow velocity, the imposed inflow condition is ramped up smoothly from a previous set value to a target value to minimize the effect of abrupt inflow acceleration.

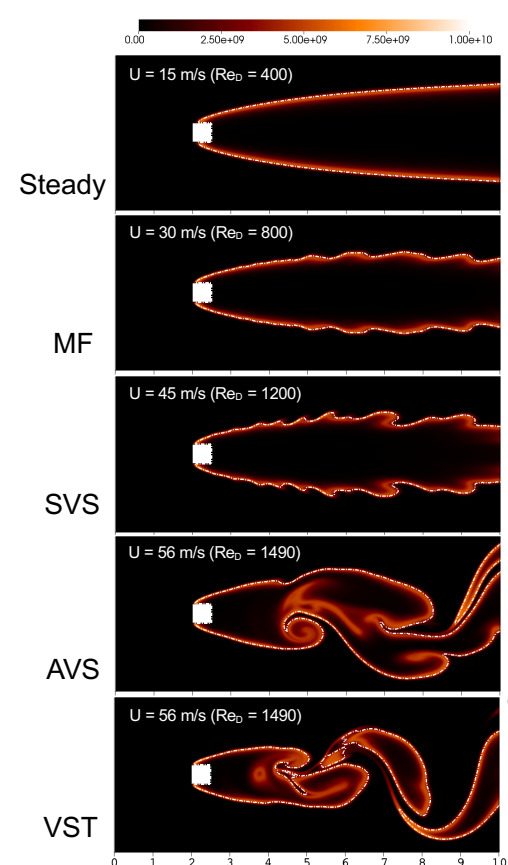

(a)

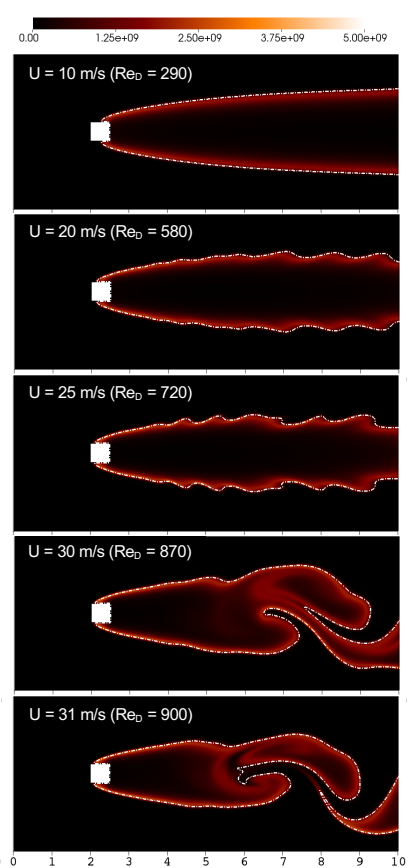

(b)

Fig. 2: Instantaneous snapshots of heat release rate as increasing the mean inflow velocity $(U)$ from steadily stable to VST, overlaid iso-contour dotted line of progress variable $(c)$ at 0.5. (a) H1U and (b) S1U. 


\section{Results}

In the following sub-sections, how unity Lewis number affects phenomenological observation of flame modes as approaching blow-off limit is to be addressed first. Secondly, how unity Lewis number affects the shear layer and mixing layer instabilities will be discussed. Thirdly, how unity Lewis number affects flame responses and instability characteristics in stronger hydrodynamic instability by decreasing density ratio (preheating inflow temperature) will be discussed. Finally, how physical mechanisms are affected by changing Lewis number from non-unity to unity and the correlated time scale analysis will be discussed.

\subsection{Response of flames with unity Lewis number}

Phenomenological observations of flame dynamics are carried out by increasing the mean inflow velocity $(U)$ until flame completely blows off. As in our previous studies [11,12], five distinct flame modes are identified as shown in Figure 2 for both hydrogen-air (H1U) and syngas-air (S1U) flames with $U$ and corresponding Reynolds number $\left(\operatorname{Re}_{D}\right)$ based on the bluff-body size $(D)$. As $U$ is increased, flames are steadily stable (Steady) at the low $U$, small-scale mild fluctuation (MF) on the shear layer, symmetric vortex shedding (SVS), asymmetric vortex shedding (AVS), and vortex street (VST). Flames are steadily anchored on the sides of the bluffbody at low velocities, and then anchoring point moves toward the leading edge as $U$ increases. As $U$ is increased further, the flames undergo unsteady fluctuations. MF is the first stage in the development of the subsequent modes of instabilities over the entire velocities. MF remains showing up on the shear layer, and the more
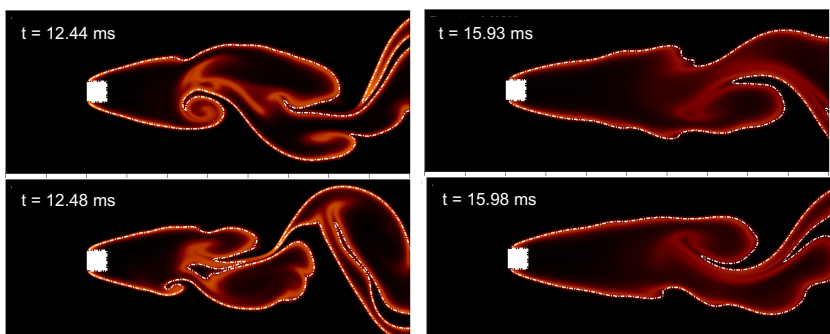

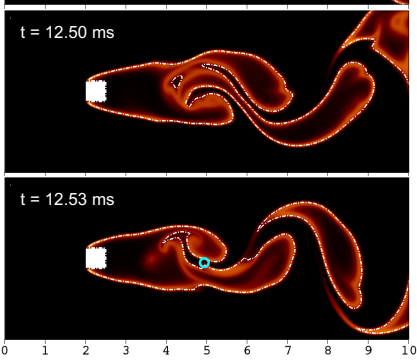

(a)

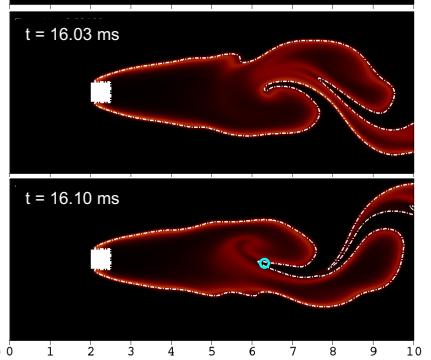

(b)

Fig. 3: Instantaneous snapshots of heat release rate for (a) $\mathrm{H} 1 \mathrm{U}$ at $U=56 \mathrm{~m} / \mathrm{s}$ and (b) $\mathrm{S} 1 \mathrm{U}$ at $U=30 \mathrm{~m} / \mathrm{s}$, showing periodic lateral fluctuations and the onset of local extinction. Iso-contour dotted line of progress variable $(c)$ at 0.5 is overlaid. 
considerable symmetric vortex shedding, so-called SVS, starts to be developed in the downstream at higher $U$.

At higher velocity conditions approaching the blow-off limit, cyclic transitions of SVS-AVS-VST are commonly observed. The vortex shedding is generated from each side asymmetrically (AVS), while the hot product gas recirculation zone immediately behind a bluff-body remains intact, as shown in the top three snapshots of Figure 3. As the blow-off limit is reached, the distance between the two flame wings become narrower, while the amplitude of the lateral fluctuation of asymmetric shedding is enhanced. In the meantime, the onset of local extinction occurs in between the asymmetric shedding vortices, which is denoted by open circles in the fourth snapshot of Figure 3, and the detached vortical flame pocket is convected downstream showing a vortex street pattern (VST). During the VST, the interaction between a hot product gas pocket and the entrainment of the fresh mixture due to the lateral fluctuations around the local extinction area may lead to re-ignition and further recovery of the attached flames toward the downstream, thus returning to SVS in the cycle. As such, the flames are sustainable in the limit cycle of SVS-AVS-VST due to the flow and chemical interactions, even at significantly high inflow velocities. Figure 4 shows the eventual blow-off events for both hydrogen-air and syngas-air flames. At the blow-off limit, after a few SVS-AVSVST cycles, the flames fail to recover from VST to SVS, and the detached flame segments are swept toward the downstream, while the remaining flames attached to the bluff-body gradually shrinks to complete extinction.

For the unity Lewis number cases, the density ratio was reduced by preheating inflow temperature (H3U or S3U) in order to examine the hydrodynamic effects. The snapshot images are shown in Figure 5. The overall development of flame

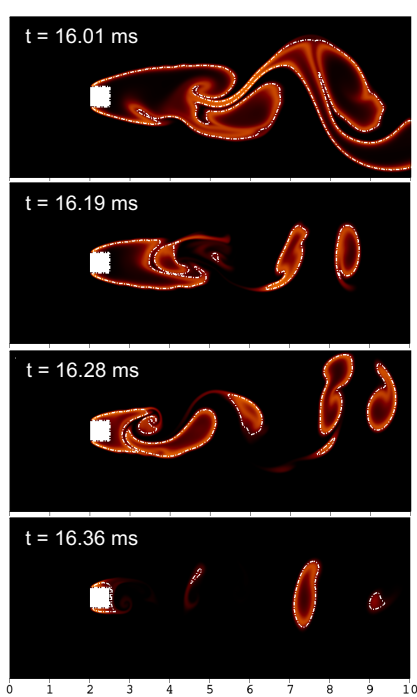

(a)

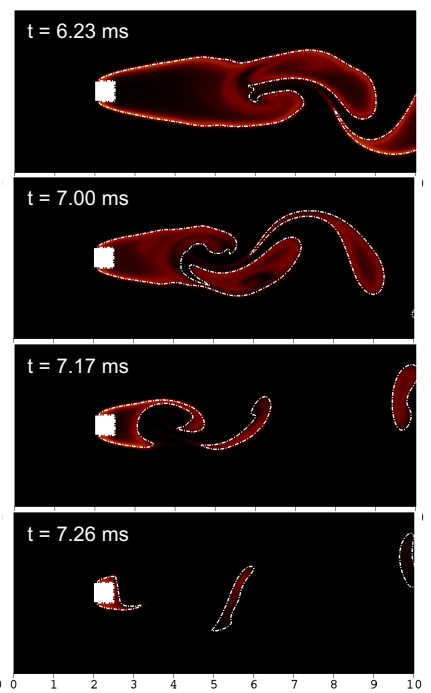

(b)

Fig. 4: Instantaneous snapshots of heat release rate showing blow-off events for (a) $\mathrm{H} 1 \mathrm{U}$ at $U$ $=58 \mathrm{~m} / \mathrm{s}$ and (b) $\mathrm{S} 1 \mathrm{U}$ at $U=31 \mathrm{~m} / \mathrm{s}$. Iso-contour dotted line of progress variable $(c)$ at 0.5 is overlaid. 

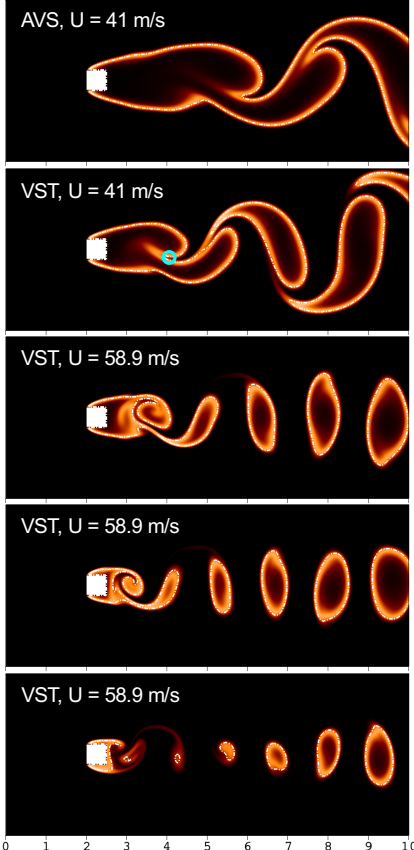

(a)

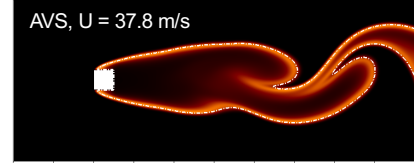

VST, $\mathrm{U}=37.8 \mathrm{~m} / \mathrm{s}$
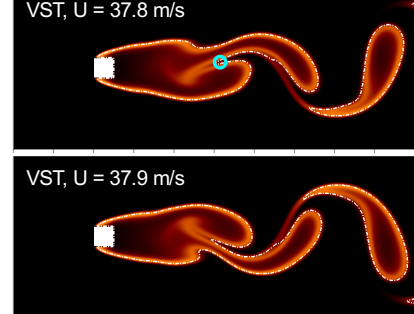

VST, $\mathrm{U}=37.9 \mathrm{~m} / \mathrm{s}$

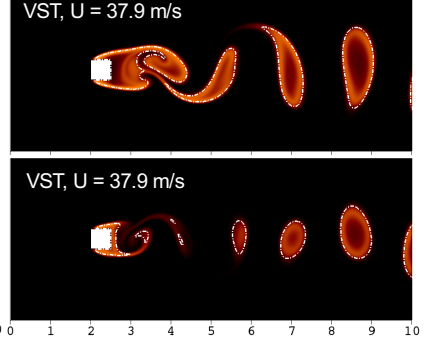

(b)

Fig. 5: Instantaneous snapshots of heat release rate showing blow-off events for (a) H3U and (b) S3U. Iso-contour dotted line of progress variable $(c)$ at 0.5 is overlaid.

responses is found to be consistent with that reported the previous study [12] for detailed transport models. For the unity Lewis number, however, symmetric flame responses such as MF and SVS disappear completely and the flame exhibits an enhanced level of the sinuous modes such as AVS and VST, as expected for the reduced density ratio. Therefore, the cyclic SVS-AVS-VST behavior is significantly suppressed.

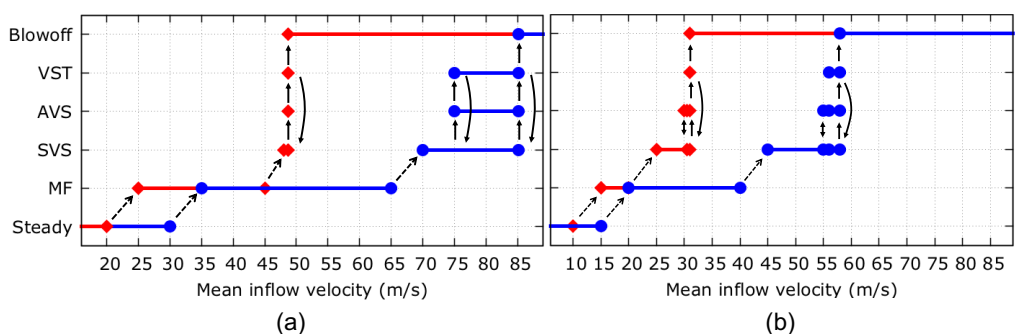

Fig. 6: Sequence diagrams of flame modes as a function of mean inflow velocity $(U)$ for both (a) non-unity [11] and (b) unity Lewis number. Hydrogen-air flames (H1 and H1U) are marked with blue circle symbols, and syngas-air flames (S1 and S1U) are marked with red diamond symbols. 


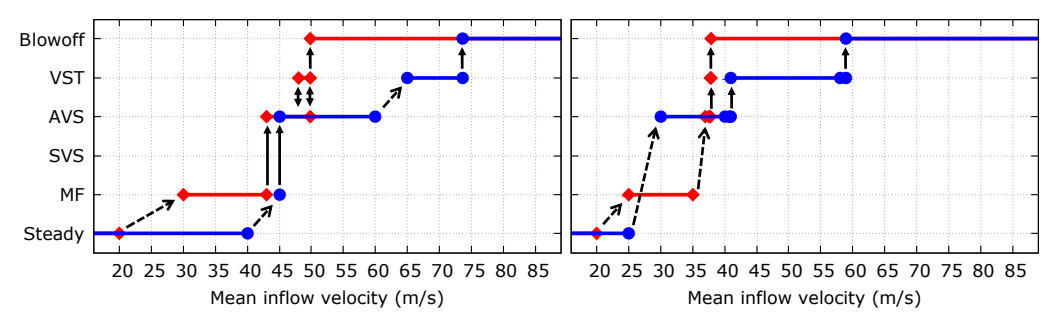

(a)

(b)

Fig. 7: Sequence diagrams of flame modes as a function of mean inflow velocity $(U)$ for both (a) non-unity [12] and (b) unity Lewis number. Hydrogen-air flames (H3 and H3U) are marked with blue circle symbols, and syngas-air flames (S3 and S3U) are marked with red diamond symbols.

The evolution of flame pattern changes as a function of $U$ is summarized in Figure 6 and 7 for both non-unity Lewis number $[11,12]$ and unity Lewis number cases. The lines with circle and diamond symbols denote hydrogen-air and syngasair mixture, respectively. The overall flame instabilities develop monotonically as $U$ is increased until blow-off. Near the blow-off limit, the cyclic SVS-AVS-VST transitions are observed for both mixtures. The velocity at which the onset of local extinction was observed $\left(U_{\text {onset }}\right)$ are at $75 \mathrm{~m} / \mathrm{s}$ for $\mathrm{H} 1,48.7 \mathrm{~m} / \mathrm{s}$ for S1, 56 $\mathrm{m} / \mathrm{s}$ for $\mathrm{H} 1 \mathrm{U}$, and $30 \mathrm{~m} / \mathrm{s}$ for $\mathrm{S} 1 \mathrm{U}$, as shown in Fig. 6 for case 1 . For case 3 shown in Fig. $7, U_{\text {onset }}$ are at $65 \mathrm{~m} / \mathrm{s}$ for $\mathrm{H} 3,48 \mathrm{~m} / \mathrm{s}$ for $\mathrm{S} 3,48 \mathrm{~m} / \mathrm{s}$ for H3U, and 37.8 $\mathrm{m} / \mathrm{s}$ for S3U. The onset of local extinction corresponds to the first point of VST in the diagrams, while for S1U it was only observed at AVS. The categorized flame modes and overall development of flame modes are similar with the previous studies [11,12] for both case 1 and 3. For case 3 shown in Fig 7, the SVS-AVSVST limit-cycle behavior observed in case 1 is suppressed to show only a single mode near blow-off limit. A key distinct finding in the present study is that $U$ of the onset of instability for Le $=1$ case is reduced compared to that of Le $<1$ case. In particular, for case 1 shown in Fig. 6, the velocity range from the onset of local extinction to the blow-off limit $\left(\Delta U=U_{\text {onset }}-U_{\text {blowoff }}\right)$ is reduced. This is because of the lower and moderate density ratio combined with the lower flow velocities for Le $=1$ cases compared to Le $<1$ cases. The detailed discussion will be provided in the next sub-section.

\subsection{Instability characteristics: Le $<1$ versus Le $=1$}

Complex flame dynamics in symmetric and asymmetric vortex sheddings under hydrodynamic instability were observed in previous studies $[11,12]$. Due to the presence of hydrogen, it is expected that local enrichment of high diffusivity fuel in the recirculation zone leads to an enhanced flame stability as reported in a recent study [7]. To assess the extent of the differential diffusion effect, the unity Lewis number flame cases are compared. Our hypothesis is that the absence of differential diffusion leads to a locally leaner condition in the recirculation zone to promote blow-off, while the small-scale diffusive-thermal instability associated with hydrogen is suppressed to yield a smoother flame topology. On the other hand, the lack of differential diffusion also leads to a lower temperature in the 

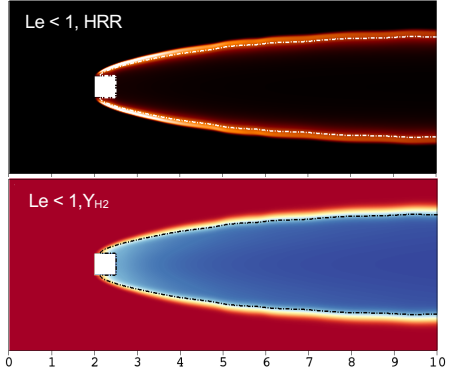

(a)
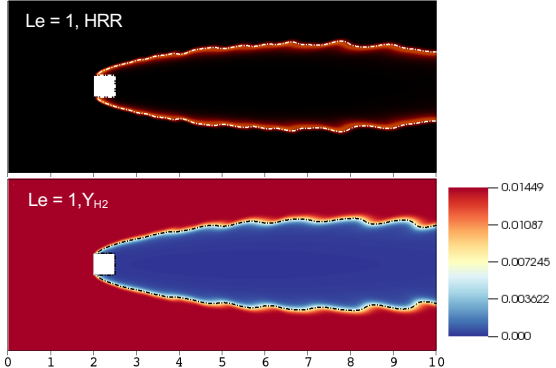

(b)

Fig. 8: Instantaneous snapshots of hydrogen-air at $U=35 \mathrm{~m} / \mathrm{s}$ for (a) H1 [11] and (b) H1U, showing mild fluctuations. Iso-contour dotted line of progress variable $(c)$ at 0.5 is overlaid.

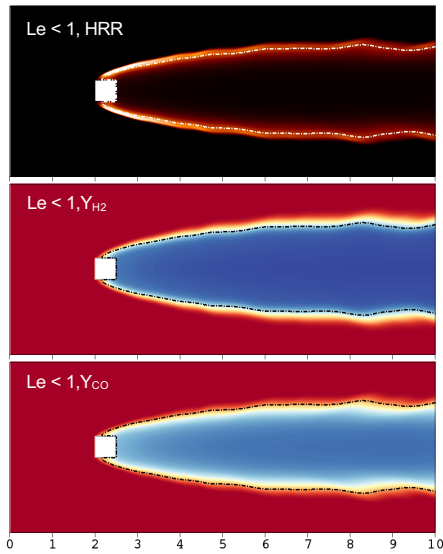

(a)

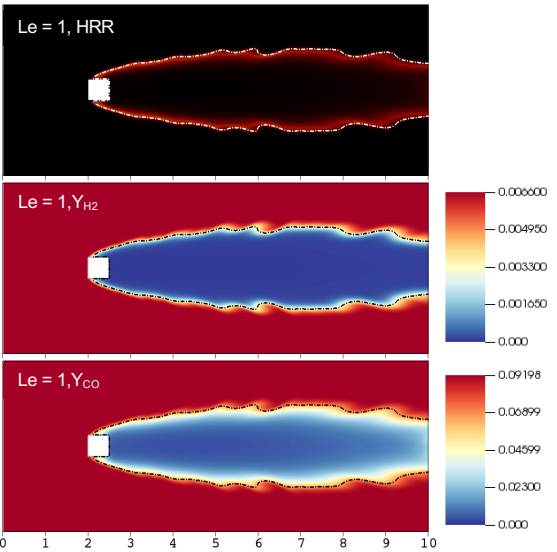

(b)

Fig. 9: Instantaneous snapshots of syngas-air at $U=25 \mathrm{~m} / \mathrm{s}$ for (a) $\mathrm{S} 1$ [11] and (b) $\mathrm{S} 1 \mathrm{U}$, showing mild fluctuations. Iso-contour dotted line of progress variable $(c)$ at 0.5 is overlaid.

recirculation zone, thereby modifying the density ratio and the resulting hydrodynamic vortex shedding behavior. In this section, comparison of non-unity and unity Lewis number flames will be made in terms of (a) the small-scale fluctuations on the upstream flame surfaces, (b) the level of lateral fluctuations of the flames downstream, and (c) the ultimate blow-off characteristics.

Figure 8 and 9 show the instantaneous snapshots of heat release rate and fuel mass fraction for $\mathrm{H} 1$ vs. $\mathrm{H} 1 \mathrm{U}$ at $U=35 \mathrm{~m} / \mathrm{s}$ and $\mathrm{S} 1$ vs. S1U at $U=25$ $\mathrm{m} / \mathrm{s}$, respectively. For each fuel, at the same $U$ the small scale fluctuations are more pronounced for the unity Lewis number cases. Figure 10 shows the spatial evolutions of temperature and species mole fractions along the center line $(y=0)$. It is evident that the local enrichment of fuel behind a bluff-body is suppressed for the Le $=1$ case, showing uniform temperature and composition profiles. The maximum flame temperature, density ratio, and frequency of MF are summarized in Table 3. The Le $=1$ flames have lower flame temperature and density ratio, closer to those for the adiabatic one-dimensional freely propagating premixed flame 


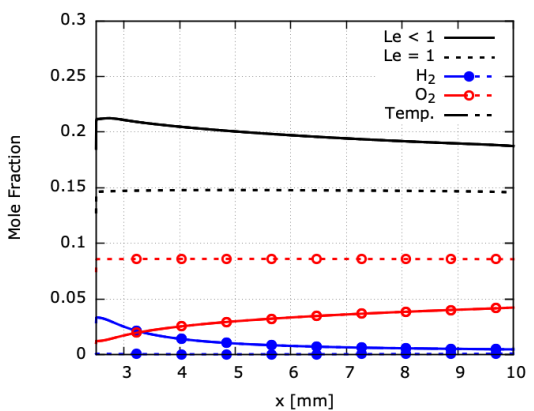

(a)

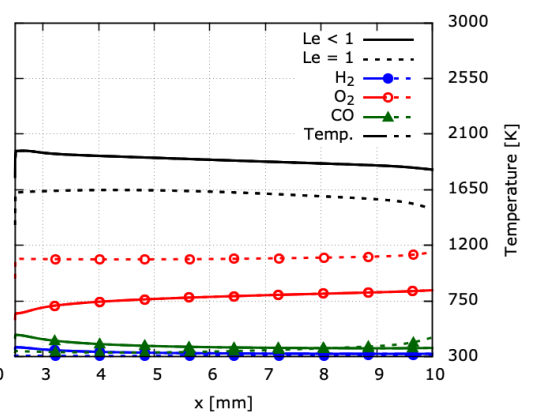

(b)

Fig. 10: Spatial evolutions of temperature and species mole fractions along the centerline (y $=0$ ) for both non-unity [11] (lines) and unity (dotted lines) Lewis number flames in (a) H1 and $\mathrm{H} 1 \mathrm{U}$ and (b) S1 and S1U.

Table 3: Scale quantities for lean premixed hydrogen-air and syngas-air flames showing smallscale instabilities on the shear layer for case $1\left(T_{\mathrm{in}}=300 \mathrm{~K}\right)$.

\begin{tabular}{c|c|c|c|c}
\hline Mixture & \multicolumn{2}{|c|}{ Hydrogen-air } & \multicolumn{2}{c}{ Syngas-air } \\
\hline Case & $\mathrm{H} 1$ & $\mathrm{H} 1 \mathrm{U}$ & $\mathrm{S} 1$ & $\mathrm{~S} 1 \mathrm{U}$ \\
\hline$U[\mathrm{~m} / \mathrm{s}]$ & 35.0 & 35.0 & 25.0 & 25.0 \\
$T_{\max }[\mathrm{K}]$ & 2123 & 1631 & 1965 & 1648 \\
$\rho_{u} / \rho_{b}(\mathrm{MF})$ & 7.27 & 4.95 & 6.23 & 5.01 \\
$f_{\mathrm{MF}}[\mathrm{kHz}]$ & 21.9 & 35.0 & 33.0 & 40.0 \\
\hline
\end{tabular}

due to the absence of the differential diffusion. These results suggest that the fluctuations of the upstream flame fronts, referred to as MF, are mainly attributed to the hydrodynamic (shear layer) instabilities which depend rather on the density ratio, which is the lower for the Le $=1$ flame due to the lower flame temperature. As further evidence, the frequency of $\mathrm{MF}\left(f_{\mathrm{MF}}\right)$ is found to be proportional to the corresponding laminar flame speed. For example, the ratio for the flame speed and the frequency between $\mathrm{H} 1$ and $\mathrm{H} 1 \mathrm{U}, S_{\mathrm{L}, \mathrm{H} 1 \mathrm{U}} / S_{\mathrm{L}, \mathrm{H} 1}$ and $f_{\mathrm{MF}, \mathrm{H} 1 \mathrm{U}} / f_{\mathrm{MF}, \mathrm{H} 1}$ are found to be identical at 1.6. Likewise, $S_{\mathrm{L}, \mathrm{S} 1 \mathrm{U}} / S_{\mathrm{L}, \mathrm{S} 1}$ and $f_{\mathrm{MF}, \mathrm{S} 1 \mathrm{U}} / f_{\mathrm{MF}, \mathrm{S} 1}$ are both found to be at 1.2 . For case 3 , since the reduced density ratio promoted asymmetric fluctuations in the overall responses, even the Le $=1$ cases do not reveal noticeable MF levels, thus the comparison is difficult.

A similar set of comparison between Le $<1$ and Le $=1$ flames is carried out to investigate how differential diffusion affects the larger level of fluctuations (AVS and VST) in mixing layer at $U_{\text {onset }}$, the onset of local extinction. Figure 3 shows two periods of lateral fluctuations in asymmetric shedding (AVS and VST) for H1U and S1U. The AVS and VST in the mixing layer that are known to be controlled by hydrodynamics in the previous study [12]. Therefore, the lateral fluctuations are scaled by the flow parameters such as $U$ and flow residence time, which is the time for a fluid particle travelling from the leading edge of the bluff-body to the local extinction point. The corresponding scale quantities are summarized in 
Table 4: Scale quantities for lean premixed hydrogen-air and syngas-air flames showing the asymmetric vortex shedding in the mixing layer for case $1\left(T_{\mathrm{in}}=300 \mathrm{~K}\right)$.

\begin{tabular}{c|c|c|c|c}
\hline Mixture & \multicolumn{2}{|c|}{ Hydrogen-air } & \multicolumn{2}{c}{ Syngas-air } \\
\hline Case & H1 & H1U & S1 & S1U \\
\hline$U_{\text {onset }}[\mathrm{m} / \mathrm{s}]$ & 75.0 & 56.0 & 48.7 & 30.0 \\
$\rho_{u} / \rho_{b}(\mathrm{VST})$ & 5.54 & 4.89 & 5.25 & 5.00 \\
$f_{\text {res }}[\mathrm{kHz}]$ & 25.0 & 20.0 & 16.0 & 7.0 \\
$f_{\text {VST }}[\mathrm{kHz}]$ & 25.0 & 16.7 & 16.4 & 7.5 \\
\hline
\end{tabular}

Table 5: Scale quantities for lean premixed hydrogen-air and syngas-air flames showing the asymmetric vortex shedding in the mixing layer for case $3\left(T_{\text {in }}=500 \mathrm{~K}\right)$.

\begin{tabular}{c|c|c|c|c}
\hline Mixture & \multicolumn{2}{|c|}{ Hydrogen-air } & \multicolumn{2}{c}{ Syngas-air } \\
\hline Case & H3 & H3U & S3 & S3U \\
\hline$U_{\text {onset }}[\mathrm{m} / \mathrm{s}]$ & 65.0 & 41.0 & 48.0 & 37.8 \\
$\rho_{u} / \rho_{b}(\mathrm{VST})$ & 2.58 & 2.98 & 3.28 & 3.16 \\
$f_{\text {res }}[\mathrm{kHz}]$ & 58.8 & 22.7 & 14.3 & 12.8 \\
$f_{\text {VST }}[\mathrm{kHz}]$ & 25.0 & 16.7 & 14.3 & 12.5 \\
\hline
\end{tabular}

Table 4 and 5 for cases 1 and 3, respectively. For both cases, the frequency of $\operatorname{VST}\left(f_{\mathrm{VST}}\right)$ is associated with $U$ and the inverse of the flow residence time $\left(f_{\text {res }}\right)$. For example, the ratio for $U$ between Le $<1$ and Le $=1$ (Case 1 vs. Case $1 \mathrm{U}$ or Case 3 vs. Case $3 \mathrm{U}$ ) is consistent with the ratio for the frequency of VST and the flow residence time. Both Le $<1$ and Le $=1$ flames are observed in the similar topological flame modes as expected from the similar density ratio. However, the AVS and VST appear at a lower $U$ due to its lower flame temperature, thereby decreasing the density ratio. Therefore, there is no noticeable effect of Le $=1$ on the flame topology in the lateral fluctuations, but the onset of mixing layer instability appears at a lower $U$ in Le $=1$ cases.

In our previous studies $[11,12]$, the blow-off mechanism was found to be dictated by the combined effects of the hot product gas pocket entrained into the recirculation zone and the ability to re-ignite the mixture within the given residence time corresponding to the lateral flame fluctuations. A larger level of lateral fluctuations help sustain the combustion at high inflow velocity conditions by facilitating the product gas entrainment into the extinguished mixture pocket. As such, it was concluded that the re-ignition of the local extinction pockets was the key mechanism controlling the overall blow-off limit, consistent with the theory by Zukoski and Marble [17]. In the present study of Le $=1$ case, although the same level of lateral fluctuations and the isolated product pocket exist, the re-ignition behavior is much suppressed due to both a decreased density ratio resulting in lateral fluctuations at lower velocities and a lower flame temperature due to the lack of preferential diffusion of hydrogen. Figure 11 shows temporal evolutions of temperature and species mole fractions at the local extinction point for a time period of $4 \mathrm{~ms}$, showing the local extinction and re-ignition phenomena for (a) H1, (b) H1U, (c) S1, and (d) S1U. Figure 12 shows similar temporal evolutions for the 

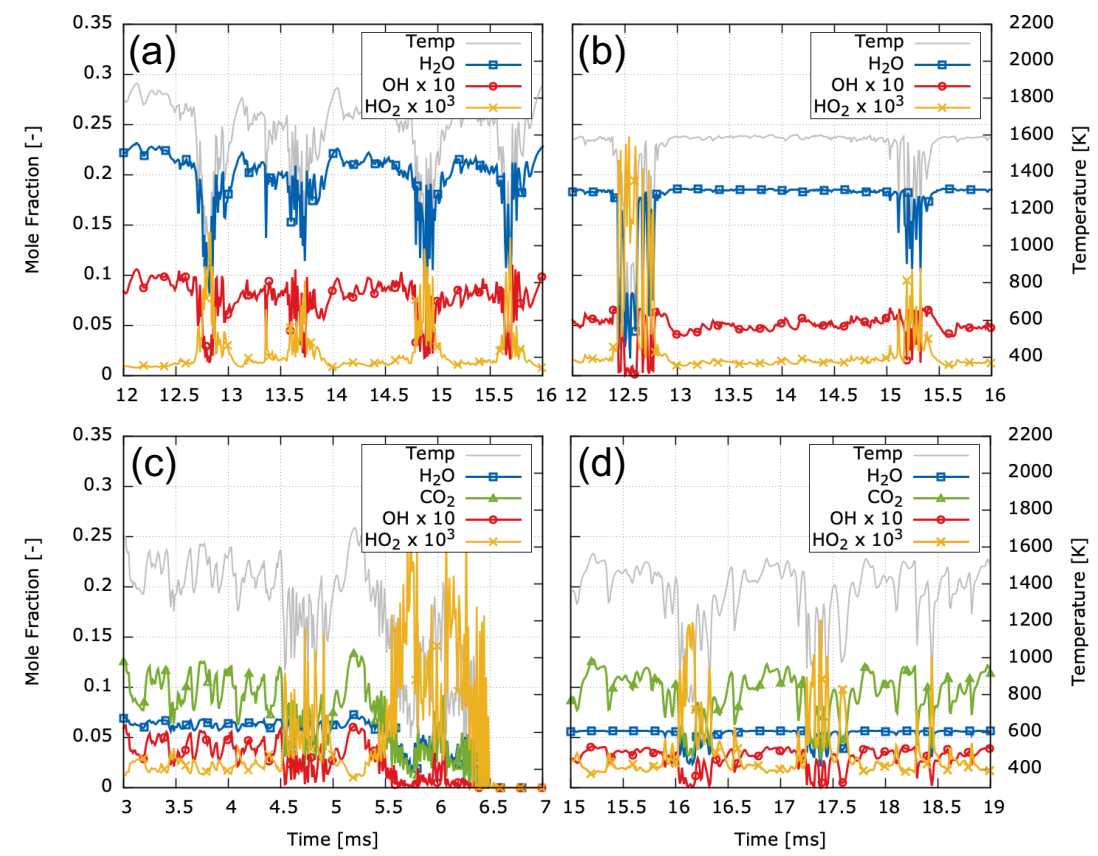

Fig. 11: Temporal evolutions of temperature and species mole fractions at the local extinction point for case $1\left(T_{\mathrm{in}}=300 \mathrm{~K}\right)$ : (a) H1 [11] at $U=75 \mathrm{~m} / \mathrm{s}$, (b) H1U at $U=56 \mathrm{~m} / \mathrm{s}$, (c) S1 [11] at $U=48.7 \mathrm{~m} / \mathrm{s}$, and (d) S1U at $U=30 \mathrm{~m} / \mathrm{s}$.

time period of $1.75 \mathrm{~ms}$ in (a) H3 and (b) H3U and $3.5 \mathrm{~ms}$ in (c) S3 and (d) S3U. In both Fig. 11 and 12, an increase in temperature or a peak in $\mathrm{HO}_{2}$ represents an re-ignition event. For S1 (Fig. 11(c)), there is only one occurrence of re-ignition after the onset of local extinction since the blow-off immediately follows due to the failure of re-ignition. Le $=1$ flames $(b, d)$ clearly show lower temperatures and lower frequencies of re-ignition events due to the lower inflow velocity (slower flow residence time). The contrasts between Le $<1$ and Le $=1$ is more pronounced in hydrogen-air flames as the flame temperature is affected more by the preferential diffusion and the ignition of hydrogen mixture is more sensitive to temperature. Such a "slow-down" of chemical activities explains why re-ignition no longer serves as a controlling mechanism for the blow-off for the Le $=1$ flames.

\subsection{Time scale analysis}

Following our previous studies $[11,12,26,27]$, the Damköhler number (Da) scaling is used in identifying the blow-off criterion. Different definitions of times scales and Da are computed as summarized in Table 6 and 7 for cases 1 and 3, respectively. For each case, the first column of numbers represents the quantities at the onset of local extinction $\left(U_{\text {onset }}\right)$ and the second column is at the blow-off limit $\left(U_{\text {blowoff }}\right)$. In Table 6, S1 has only one column due to the fact that the blow-off occurs followed by the onset of local extinction. The choices of velocity conditions in Tables 6 and 7 follow Figures 6 and 7, respectively. 

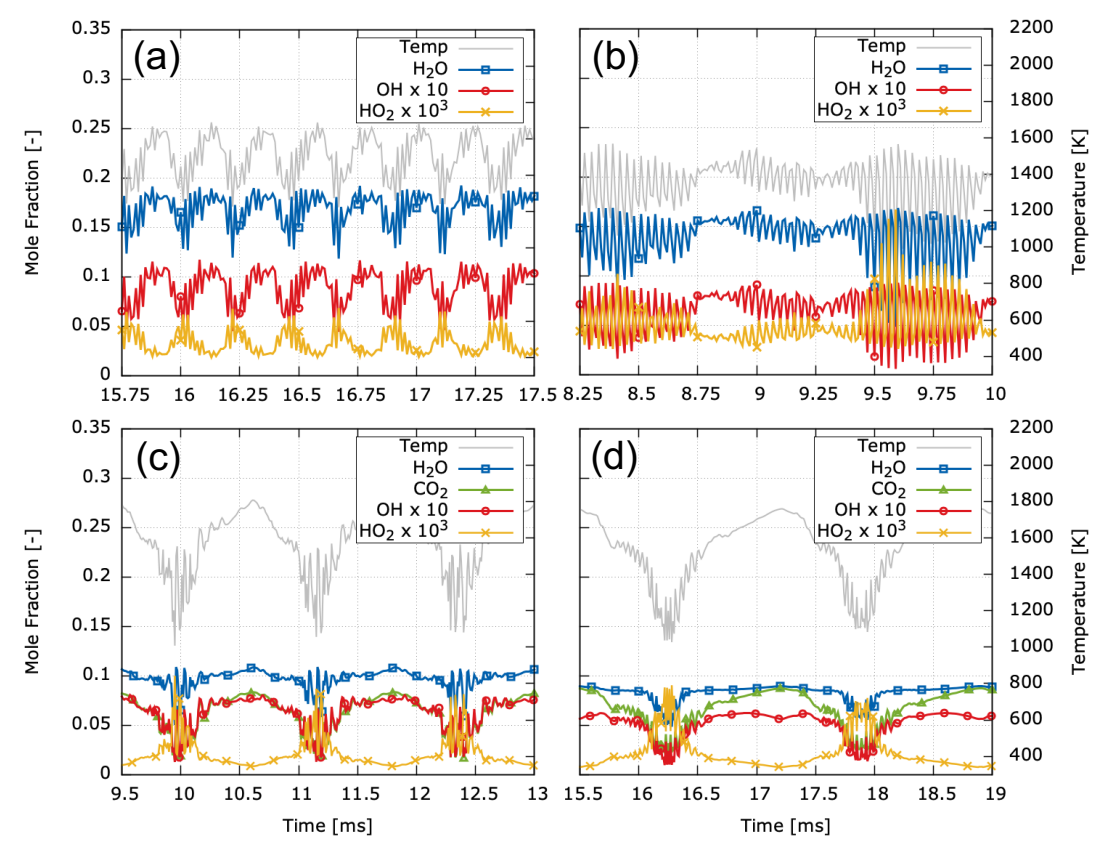

Fig. 12: Temporal evolutions of temperature and species mole fractions at the local extinction point for case $3\left(T_{\mathrm{in}}=500 \mathrm{~K}\right)$ : (a) H3 [12] at $U=65 \mathrm{~m} / \mathrm{s}$, (b) H3U at $U=41 \mathrm{~m} / \mathrm{s}$, (c) S3 [12] at $U=48 \mathrm{~m} / \mathrm{s}$, and (d) S3U at $U=37.8 \mathrm{~m} / \mathrm{s}$.

Since Da is defined as the ratio of flow and chemical time scales, two flow time scales and two chemical time scales are considered. The flow time scale was defined by (1) the recirculation zone size from the leading edge $\left(x_{\text {ext }}\right)$ over the inflow velocity $(U), \tau_{\text {res }}$, or by (2) the lateral fluctuation time (inverse of frequency), $\tau_{\text {lateral }}$. For the chemical time scales, (1) the ignition delay time, $\tau_{\text {ig }}$, was calculated by the auto-ignition of the corresponding homogeneous mixture at the initial temperature at the local extinction point, and (2) the nominal flame time scale, $\tau_{\text {flame}}$, defined as the nominal flame thickness $\left(\alpha / S_{\mathrm{L}}\right)$ divided by the corresponding laminar flame speed $\left(S_{\mathrm{L}}\right)$. This yields a total of four Da's, two of which were based on the ignition delay time, $\mathrm{Da}_{\mathrm{ig}}$, and the other two based on the flame time, Daflame.

For Le $=1$ cases $($ case $1 \mathrm{U}$ and $3 \mathrm{U})$, the density ratios $\left(\rho_{u} / \rho_{b(\mathrm{VST})}\right)$ as shown in both Tables 6 and 7 are nearly identical to those at the corresponding Le $<1$ cases, even though $U_{\text {onset }}$ and $U_{\text {blowoff }}$ change significantly. This confirms that the lateral fluctuations and vortex shedding (AVS, VST) modes are driven by fluid dynamics.

In our previous studies $[11,12]$ with detailed transport models (H1, S1, H3, and S3), it was found that Daig predicts the blow-off behavior consistently at an $O(1)$ magnitude. Therefore, it was concluded that the re-ignition characteristics control the blow-off mechanism. Considering the complementary Le $=1$ cases ( $\mathrm{H} 1 \mathrm{U}, \mathrm{S} 1 \mathrm{U}, \mathrm{H} 3 \mathrm{U}$, and $\mathrm{S} 3 \mathrm{U})$, however, the conclusion is not subjected to further scrutiny as it appears that Daflame being $O(1)$ in magnitude serves as a better indicator to predict the blow-off condition. As discussed above, this is attributed to the combined effects of (a) increased hydrodynamic instability due to the reduced 
density ratio and (b) the weakening of re-ignition due to the lower temperature at the local extinction pocket. This is the most surprising finding in the present study, as an unexpected outcome of investigating the Le $=1$ effect: the blow-off mechanism may be characterized by either a local extinction or failure of re-ignition depending on the specific parametric conditions. Further studies are needed in order to draw a conclusive answer to the question and develop a unified theory to predict the blow-off criterion.

\section{Conclusions}

High fidelity numerical simulations were conducted to investigate unity Lewis number effects on dynamics of lean hydrogen-air and syngas-air premixed flames stabilized on a meso-scale bluff-body. The unity Lewis number for all species was imposed as a means to isolate the effects of preferential diffusion on flame stabilization and blow-off dynamics. Two different inflow temperatures with unity Lewis number were applied for both fuels to examine two levels of hydrodynamic instability (density ratio), such as (a) case $1 \mathrm{U}$ of $T_{\mathrm{in}}=300 \mathrm{~K}$ and (b) case $3 \mathrm{U}$ of $T_{\text {in }}=500 \mathrm{~K}$. For a wide range of inflow velocity conditions, non-unity (Le < 1 ) and unity Lewis number $(\mathrm{Le}=1)$ cases were compared to examine how unity Lewis number (equi-diffusion) affects the overall flame responses, the shear layer, mixing layer instabilities, and blow-off mechanism.

As the mean inflow velocity approaches the blow-off limit, the five distinct flame modes were identified for the Le $=1$ cases as in the Le $<1$ cases. The

Table 6: Characteristic time scales and physical quantities for both non-unity [11] and unity Lewis number for in hydrogen-air and syngas mixtures at $\Phi=0.5$ and $T_{\mathrm{in}}=300 \mathrm{~K}$.

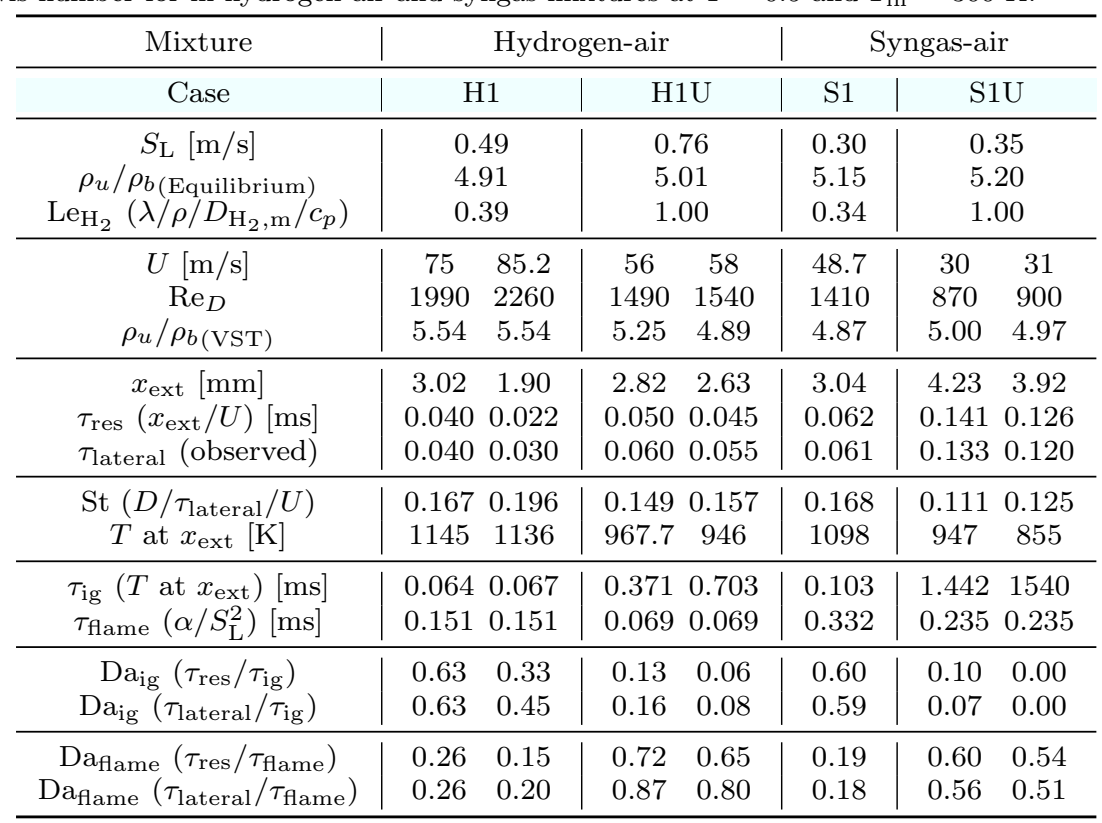


Table 7: Characteristic time scales and physical quantities for both non-unity [12] and unity Lewis number for in hydrogen-air and syngas mixtures at $\Phi=0.5$ and $T_{\mathrm{in}}=500 \mathrm{~K}$.

\begin{tabular}{|c|c|c|c|c|c|c|c|c|}
\hline Mixture & \multicolumn{4}{|c|}{ Hydrogen-air } & \multicolumn{4}{|c|}{ Syngas-air } \\
\hline Case & \multicolumn{2}{|c|}{ H3 } & \multicolumn{2}{|c|}{$\mathrm{H} 3 \mathrm{U}$} & \multicolumn{2}{|c|}{ S3 } & \multicolumn{2}{|c|}{$\mathrm{S} 3 \mathrm{U}$} \\
\hline$S_{\mathrm{L}}[\mathrm{m} / \mathrm{s}]$ & \multirow{2}{*}{\multicolumn{2}{|c|}{$\begin{array}{l}2.02 \\
3.27\end{array}$}} & \multicolumn{2}{|c|}{2.04} & \multicolumn{2}{|c|}{0.99} & \multicolumn{2}{|c|}{1.01} \\
\hline$\rho_{u} / \rho_{b(\text { Equilibrium })}$ & & & \multirow{2}{*}{\multicolumn{2}{|c|}{$\begin{array}{l}3.31 \\
1.00 \\
\end{array}$}} & \multirow{2}{*}{\multicolumn{2}{|c|}{$\begin{array}{l}3.38 \\
0.34 \\
\end{array}$}} & \multirow{2}{*}{\multicolumn{2}{|c|}{$\begin{array}{l}3.41 \\
1.00\end{array}$}} \\
\hline $\mathrm{Le}_{\mathrm{H}_{2}}\left(\lambda / \rho / D_{\mathrm{H}_{2}, \mathrm{~m}} / c_{p}\right)$ & \multicolumn{2}{|c|}{0.39} & & & & & & \\
\hline$U[\mathrm{~m} / \mathrm{s}]$ & 65 & 73.6 & 41 & 58.9 & 48 & 49.8 & 37.8 & 37.9 \\
\hline $\operatorname{Re}_{D}$ & 716 & 811 & 451 & 650 & 580 & 600 & 455 & 456 \\
\hline$\rho_{u} / \rho_{b(\mathrm{VST})}$ & 2.58 & 2.71 & 2.98 & 2.69 & 3.28 & 3.18 & 3.16 & 3.12 \\
\hline$x_{\text {ext }}[\mathrm{mm}]$ & 1.07 & 0.71 & 1.82 & 1.27 & 3.35 & 2.34 & 2.96 & 2.99 \\
\hline$\tau_{\text {res }}\left(x_{\text {ext }} / U\right)[\mathrm{ms}]$ & 0.017 & 0.010 & 0.044 & 0.022 & 0.070 & 0.047 & 0.078 & 0.079 \\
\hline$\tau_{\text {lateral }}($ observed $)$ & 0.040 & 0.026 & 0.060 & 0.035 & 0.070 & 0.050 & 0.080 & 0.080 \\
\hline $\operatorname{St}\left(D / \tau_{\text {lateral }} / U\right)$ & 0.192 & 0.261 & 0.203 & 0.242 & 0.148 & 0.201 & 0.165 & 0.165 \\
\hline$T$ at $x_{\text {ext }}[\mathrm{K}]$ & 1223 & 1180 & 1040 & 1020 & 1119 & 1125 & 974 & 995 \\
\hline$\tau_{\text {ig }}\left(T\right.$ at $\left.x_{\text {ext }}\right)[\mathrm{ms}]$ & 0.042 & 0.052 & 0.138 & 0.169 & 0.090 & 0.086 & 0.387 & 0.275 \\
\hline$\tau_{\text {flame }}\left(\alpha / S_{\mathrm{L}}^{2}\right)[\mathrm{ms}]$ & 0.021 & 0.021 & 0.021 & 0.021 & 0.071 & 0.071 & 0.068 & 0.068 \\
\hline $\operatorname{Da}_{\mathrm{ig}}\left(\tau_{\mathrm{res}} / \tau_{\mathrm{ig}}\right)$ & 0.40 & 0.19 & 0.32 & 0.13 & 0.78 & 0.55 & 0.20 & 0.29 \\
\hline $\operatorname{Da}_{\text {ig }}\left(\tau_{\text {lateral }} / \tau_{\text {ig }}\right)$ & 0.95 & 0.75 & 0.43 & 0.21 & 0.78 & 0.58 & 0.20 & 0.29 \\
\hline Daflame $\left(\tau_{\text {res }} / \tau_{\text {flame }}\right.$ & 0.81 & 0.48 & 2.10 & 1.05 & 0.98 & 0.66 & 1.15 & 1.16 \\
\hline $\operatorname{Da}_{\text {flame }}\left(\tau_{\text {lateral }} / \tau_{\text {flame }}\right)$ & 1.90 & 1.24 & 2.85 & 1.67 & 0.98 & 0.70 & 1.17 & 1.17 \\
\hline
\end{tabular}

effect of preferential diffusion was diminished at the higher inflow velocity from conditions of the onset of local extinction. In general, the unity Lewis number leads to a lower flame temperature and thus a lower density ratio between the unburned and burned gases. This results in an increased level of hydrodynamic instabilities manifested as lateral fluctuations and vortex sheddings occurring at a lower inflow velocity. The lower burned product gas temperature also leads to the fact that the re-ignition of local mixture at extinction by the product gas entrainment is weakened. The net outcome is that the Le $=1$ flames are more vulnerable to blowoff at lower inflow velocities. Furthermore, the Le $=1$ flames at conditions under study do not appear to benefit much from re-ignition events, leading to a revised physical conclusion that the ultimate blow-off behavior at high velocity conditions are mainly controlled by the onset of local extinction.

The present study demonstrated that flame blow-off dynamics are complex and intricate combinations of different physical and chemical processes, and a unified theoretical blow-off criterion awaits and deserves further investigations. Work is underway to identify and characterize distinct chemical and physical signatures of local extinction and re-ignition by utilizing the computational singular perturbation method as employed in turbulent flames [28,29]. While the present and previous studies $[11,12,26,27]$ based on two-dimensional simulations have provided understanding of key relevant physics in real systems, additional three-dimensional effects, such as the spanwise flow variations, may play an important role in flame instabilities (facilitating or impeding) and blow-off limit (extension or contraction). To investigate these issues, large scale three-dimensional simulations are in progress and will be reported in future work. 
Acknowledgements This work was sponsored by King Abdullah University of Science and Technology (KAUST). Computational resources were provided by the KAUST Supercomputing Laboratory (KSL). BJL was supported by the Korea Institute of Energy Technology Evaluation and Planning (KETEP) and the Ministry of Trade, Industry and Energy (MOTIE) of the Republic of Korea (Grant No. 20181110100290).

\section{Funding}

YJK and HGI were funded by King Abdullah University of Science and Technology (no grant number). BJL was sponsored by the Korea Institute of Energy Technology Evaluation and Planning (KETEP) and the Ministry of Trade, Industry and Energy (MOTIE) of the Republic of Korea (Grant No. 20181110100290).

\section{Conflict of Interest}

The authors declare that they have no conflict of interest.

\section{References}

1. Shanbhogue, S.J., Husain, S., Lieuwen, T.: Lean blowoff of bluff body stabilized flames: Scaling and dynamics. Prog. Energy Combust. Sci. 35(1), 98-120 (2009)

2. Suzuki, H., Inoue, Y., Nishimura, T., Fukutani, K., Suzuki, K.: Unsteady flow in a channel obstructed by a square rod (crisscross motion of vortex). Int. J. Heat Fluid Flow 14(1), 2-9 (1993)

3. Suzuki, K., Suzuki, H.: Instantaneous structure and statistical feature of unsteady flow in a channel obstructed by a square rod. Int. J. Heat Fluid Flow 15(6), 426-437 (1994)

4. Nair, S., Lieuwen, T.C.: Near-blowoff dynamics of a bluff-body stabilized flame. J. Propul. Power 23(2), 421-427 (2007)

5. Morales, A.J., Lasky, I.M., Geikie, M.K., Engelmann, C.A., Ahmed, K.A.: Mechanisms of flame extinction and lean blowout of bluff body stabilized flames. Combust. Flame 203, 31-45 (2019)

6. Kedia, K.S., Ghoniem, A.F.: The anchoring mechanism of a bluff-body stabilized laminar premixed flame. Combust. Flame 161(9), 2327-2339 (2014)

7. Vance, F., Shoshin, Y., van Oijen, J., de Goey, L.: Effect of lewis number on premixed laminar lean-limit flames stabilized on a bluff body. Proc. Combust. Inst. 37(2), 1663-1672 (2019)

8. Chaudhuri, S., Kostka, S., Renfro, M.W., Cetegen, B.M.: Blowoff dynamics of bluff body stabilized turbulent premixed flames. Combustion and Flame 157(4), 790-802 (2010)

9. Chowdhury, B.R., Cetegen, B.M.: Experimental study of the effects of free stream turbulence on characteristics and flame structure of bluff-body stabilized conical lean premixed flames. Combust. Flame 178, 311-328 (2017)

10. Barlow, R.S., Dunn, M.J., Sweeney, M.S., Hochgreb, S.: Effects of preferential transport in turbulent bluff-body-stabilized lean premixed ch4/air flames. Combust. Flame 159(8), 2563-2575 (2012)

11. Kim, Y.J., Lee, B.J., Im, H.G.: Dynamics of lean premixed flames stabilized on a mesoscale bluff-body in an unconfined flow field. Math. Model. Nat. Phenom. 13(6), 48-63 (2018)

12. Kim, Y.J., Lee, B.J., Im, H.G.: Hydrodynamic and chemical scaling for blow-off dynamics of lean premixed flames stabilized on a meso-scale bluff-body. Proc. Combust. Inst. 37(2), 1831-1841 (2019)

13. Emerson, B., O'Connor, J., Juniper, M., Lieuwen, T.: Density ratio effects on reacting bluff-body flow field characteristics. J. Fluid Mech. 706, 219-250 (2012)

14. Emerson, B., Jagtap, S., Quinlan, J.M., Renfro, M.W., Cetegen, B.M., Lieuwen, T.: Spatiotemporal linear stability analysis of stratified planar wakes: Velocity and density asymmetry effects. Phys. Fluids 28(4), 045101 (2016) 
15. Kiel, B., Garwick, K., Gord, J., Miller, J., Lynch, A., Hill, R., Phillips, S.: A detailed investigation of bluff body stabilized flames. In: 45th AIAA Aerospace Sciences Meeting and Exhibit, p. 168 (2007)

16. Pathania, R.S., Skiba, A., Sidey, J.A., Mastorakos, E.: Blow-off mechanism in a turbulent premixed bluff-body stabilized flame with pre-vaporized fuels. In: AIAA Scitech 2019 Forum, p. 2238 (2019)

17. Zukoski, E.E., Marble, F.E.: Experiments concerning the mechanism of flame blowoff from bluff bodies. Proc. Gas Dynamics Symposium Aerothermochemistry pp. 205-210 (1956)

18. Chen, J.H., Choudhary, A., de Supinski, B., DeVries, M., Hawkes, E.R., Klasky, S., Liao, W.K., Ma, K.L., Mellor-Crummey, J., Podhorszki, N., Sankaran, R., Shende, S., Yoo, C.S.: Terascale direct numerical simulations of turbulent combustion using s3d. Comput. Sci. Disc. 2(1), 015001 (2009)

19. Kim, Y.J., Lee, B.J., Im, H.G.: Near-blowoff dynamics of lean premixed flames stabilized on a meso-scale bluff body. In: 54th AIAA Aerospace Sciences Meeting, p. 0956 (2016)

20. Kee, R.J., Rupley, F.M., Miller, J.A.: CHEMKIN-II: A FORTRAN chemical kinetics package for the analysis of gas-phase chemical kinetics. Tech. Rep. SAND89-8009, Sandia National Laboratories (1989)

21. Smooke, M.D., Giovangigli, V.: Lecture Notes in Physics, vol. 384. Springer-Verlag (1991). P. $1-28$

22. Li, J., Zhao, Z., Kazakov, A., Dryer, F.L.: An updated comprehensive kinetic model of hydrogen combustion. Int. J. Chem. Kinet. 36(10), 566-575 (2004)

23. Li, J., Zhao, Z., Kazakov, A., Chaos, M., Dryer, F.L., Scire, J.J.: A comprehensive kinetic mechanism for co, ch2o, and ch3oh combustion. Int. J. Chem. Kinet. 39(3), 109-136 (2007)

24. Yoo, C.S., Wang, Y., Trouvé, A., Im, H.G.: Characteristic boundary conditions for direct simulations of turbulent counterflow flames. Combust. Theory Model. 9(4), 617-646 (2005)

25. Yoo, C.S., Im, H.G.: Characteristic boundary conditions for simulations of compressible reacting flows with multi-dimensional, viscous and reaction effects. Combust. Theory Model. 11(2), 259-286 (2007)

26. Lee, B.J., Yoo, C.S., Im, H.G.: Dynamics of bluff-body-stabilized premixed hydrogen/air flames in a narrow channel. Combust. Flame 162(6), 2602-2609 (2015)

27. Lee, B.J., Im, H.G.: Dynamics of bluff-body-stabilized lean premixed syngas flames in a meso-scale channel. Proc. Combust. Inst. 36(1), 1569-1576 (2017)

28. Manias, D.M., Tingas, E.A., Pérez, F.E.H., Galassi, R.M., Ciottoli, P.P., Valorani, M., Im, H.G.: Investigation of the turbulent flame structure and topology at different karlovitz numbers using the tangential stretching rate index. Combust. Flame 200, 155-167 (2019)

29. Manias, D.M., Tingas, E.A., Minamoto, Y., Im, H.G.: Topological and chemical characteristics of turbulent flames at mild conditions. Combust. Flame 208, 86-98 (2019) 